\title{
Son las cosas pequeñas las que cuentan: El uso del diminutivo en la tradición oral Lenca
}

Claudia Sagrario Torres* Raquel Lobo Morales*

... A pues entonces vinieron los españoles a quitar aquellos indios y a ir ministrando a las indias para que este lugar sea más un país diferente o que haiga más rozamiento de gente ya mestiza. Aunque habemos unos que somos puru indito indito, pero que ya se va cruzando y ya es como el coyote con el chucho...

(Antonio Rodríguez, "La raza de Lempira”)

El miedo a la asimilación está presente en la anterior cita y es una realidad para los Lencas de Honduras. La cultura dominante día a día los obliga a convertirse en una fuerza laboral migratoria que sirve a los programas de "modernización del estado" de Honduras. Los gobiernos, independientemente del partido político, buscan aumentar la producción para el mercado global y toman decisiones que desintegran los espacios étnicos en Honduras. Los Lencas están muy concientes de la gran amenaza que pende sobre su comunidad y para sobrevivir responden con mecanismos de resistencia cotidiana que les asegure una cultura propia.

Un importante mecanismo para la sobre vivencia cultural es la tradición oral y en ella el uso del diminutivo que asegura resaltar los elementos más valorados por la comunidad. La entonación y el énfasis en el relato valoran ciertas ideas y creencias, mientras otros elementos pasan a un segundo plano. Entre los Lencas de Honduras, cuyo analfabetismo llega a un $60 \%$, narrar relatos sirve como una forma básica para articular una específica perspectiva cultural que difiere del paradigma dominante. Hay elementos claves que emergen de la sincrética cultura Lenca y son estructuras lingüísticas específicas en la tradición oral.

En este estudio busco demostrar una correlación definitiva entre el uso del diminutivo y entre conceptos y objetos de mucha significación en las costumbres ceremoniales del pueblo Lenca. El estudio tiene dos propósitos: primero demostrar que el diminutivo es un instrumento importante en el "performance" Lenca, y segundo, que el diminutivo sirve un propósito análogo al de un código conciente 0

\footnotetext{
*Claudia Sagrario Torres, claudinetorres7@yahoo.com

Facultad de Humanidades y Artes, Escuela de Letras. Universidad Nacional Autónoma de Honduras

*Raquel Lobo Morales, fediamondhn@yahoo.com

Facultad de Humanidades y Artes, Escuela de Letras. Universidad Nacional Autónoma de Honduras
} 
inconscientemente articulado, el cual resalta las creencias de mayor valor para la identidad Lenca. Esto es particularmente aparente cuando el diminutivo marca un ritual que ha sido reprimido en las últimas décadas.

\section{HISTORIA Y CULTURA}

Yamaranguila es una comunidad Lenca de aproximadamente 15,000 personas. Está localizada en las montañas del Departamento de Intibucá en el suroeste de Honduras. Es una región montañosa con unos pocos valles pequeños y ríos. Hasta hace unas décadas, no había carreteras y las que existían estaban en malas condiciones. Era una región aislada con su propia microeconomía de subsistencia. Está región se encuentra fuera del cinturón agroindustrial de Honduras que se encuentra hacia al Norte y el Este de Yamaranguila. Esta proximidad ha afectado a la comunidad Lenca, ya que gradualmente se están convirtiendo en protoproletarios que proveen mano de obra a las agroindustrias. Gradualmente están perdiendo sus tierras comunales debido a las nuevas leyes de la reforma agraria en Honduras. De acuerdo a estas leyes, las tierras comunales no están protegidas y entran al mercado libre y a mejor postor. Esto significa que muchas de las tierras comunales, que antes sostenían la cohesión comunitaria, son vendidas. Esta situación ha creado un gran conflicto entre los Lencas y los vecinos Ladinos (no indígenas), quienes buscan las tierras comunales y manipulan a políticos corruptos para apropiarse de las mismas.

Los Lencas han sido y son uno de los pueblos más numerosos en Honduras. Actualmente, son cerca de 150,000 distribuidos en las altas sierras del suroeste de Honduras en los Departamentos de Francisco Morazán, Comayagua, La Paz, Intibucá, Lempira, Copán y parte de Valle. En la antigüedad vivían también en los valles del Norte, pero con la llegada de los españoles en el Siglo XVI, se movieron a las regiones montañosas buscando más protección y aislándose de los conquistadores. Los Lencas fueron uno de los pueblos indígenas que más combatió contra los españoles, inclusive cuando otras regiones ya estaban completamente conquistadas en el Siglo XVII los Lencas ponían resistencia. La resistencia armada contra los españoles está muy bien documentada y no terminó hasta cerca del 1637 cuando Lempira, un cacique Lenca, fue asesinado y los Lencas se dispersaron (Chapman 1985). Muchos murieron de enfermedades y en cautiverio poco después de la conquista, cerca del $80 \%$ de la población (Becerra 1985 y Chapman 1985).

Actualmente los Lencas son campesinos que trabajan la tierra comunal, en algunos casos, y, en otros, se están convirtiendo en protoproletarios al servido de las fincas de café y de los cultivos de tomates en el cercano Valle de Comayagua. Existe muy 
poca diferencia de clases entre la comunidad Lenca porque quienes han adquirido ventajas económicas han abandonado la comunidad y se identifican más con la comunidad ladina. Estas ventajas económicas, en muchos casos, han ocurrido por participar en el ejército. Los reclutamientos a la fuerza los llevan lejos y luego no regresan a sus hogares. Muchos abandonan a sus mujeres e hijos y rehacen su vida con otra pareja. Aquellos que logran regresar sienten una fuerte antipatía por el ejército y por la cultura mestiza o ladina.

Según Richard Lyle Campbell, él grabó al último hablante lenca en 1970. Otros autores sospechaban que todavía quedaban hablantes en 1976, sin embargo, la Carrera de Letras investigó de 1978 hasta 1982 sin encontrar hablantes. Los lencas hablan español con su propia variante dialectal que se caracteriza por: (a) uso extensivo del diminutivo, (b) cambio de la o por u, (c) cambio de la e por i, (d) uso de contracciones, (e) uso de términos lencas, (f) uso de arcaísmos, (g) presencia de la [s], (h) ausencia de una concordancia por género, (i) pronunciación de la $h$ velarizada, y (j) creación de verbos a partir de los sustantivos. Esta forma dialectal caracteriza la zona y existen otros rasgos culturales que caracterizan esta etnía. (Carías y otros 1988, Herranz 1987).

Yamaranguila es una de las comunidades lencas que, según Campbell (1976), mantiene las prácticas de la cultura lenca. Celebran el Guancasco, la Carrera de Patos, la Carrera de Cintas, las Composturas, el Pago a la Tierra, la veneración por los lugares altos, practican medicina tradicional, la mano vuelta (trabajo por reciprocidad o trueque), La Alcaldía de la Vara Alta, visten ropa tradicional (sobretodo las mujeres), practican métodos agrícolas como el uso del pujaguante, comen comida tradicional (ticucos o tamal de viaje), y participan en la tradición oral a través de autores que cuentan las historias. Las autoridades cultivan el respeto de la comunidad a través de la participación en el trabajo comunitario y al compartir con toda la comunidad los beneficios obtenidos.

El Guancasco es una de las ceremonias culturales más importantes. Ha existido desde épocas precolombinas cuando los diferentes ciudades de los lencas no controlaban la política hegemónica y se mantenían en constantes guerras entre unos y otros (Chapman 1985). Estas comunidades guerreras eran ciudades estados con autonomía y requerían de los tratados de paz para mantener una estabilidad política. Estos tratados eran los guancascos que permitían crear alianzas para enfrentar a otros grupos y evitar la constante pérdida de vidas. Más tarde, cuando los españoles conquistaron la región y la iglesia estableció una mayor presión, los guancascos incorporaron los elementos católicos y se convirtieron en celebraciones para los patrones de las comunidades. En el caso de 
Yamaranguila, su guancasco se celebra con el cercano pueblo de Intibucá. San Antonio es el santo patrón de Yamaranguila y la Virgen de la Candelaria es la patrona de Intibucá.

La Carrera de Patos y la de Cintas son en origen europeas, pero se han adaptado para abrir espacios para el cortejo. Las mujeres en edad de casarse colocan sus cintas sobre una cuerda alta estirada entre dos postes y los jóvenes deben arrancarlas de la cuerda mientras cabalgan a gran rapidez. La dueña de la cinta arrancada, debe bailar en una fiesta posterior con el poseedor de la misma. En algunas Carreras guindan patos en vez de cintas. Esta carrera es más difícil, puesto que los patos oscilan en la cuerda y resulta mucho más difícil agarrarles el cuello y bajar el pato. Esta carrera dura horas y los jinetes terminan con las manos ensangrentadas y heridas.

Las Composturas y los Pagos a la Tierra son ceremonias sincréticas con oraciones católicas y con rituales precolombinos. Básicamente, corresponden a las antiguas ofrendas mayas para los dioses en señal de agradecimiento por los bienes recibidos por los humanos. Según León Portilla (1988), el pensamiento maya determina que los humanos no reciben nada gratis, se debe ofrendar a los dioses para recibir. Y al ofrendar y agradecer a los dioses, éstos se aseguran de existir. Poseen un sentido de reciprocidad entre lo humano y lo divino. Con estas ceremonias, los lencas ofrecían a sus dioses bienes a cambio de favores divinos. La tierra produce porque los lencas le ofrecen algo a cambio. El Pago a la Tierra es una ofrenda en que el humano sacrifica pavos y gallinas, su sangre es vertida en "el ombligo de la tierra". Se escarba un hoyito en la tierra frente al altar en el cual se echa la sangre del animal recién sacrificado. La Tierra requiere nutrientes para asegurar una buena cosecha de maíz, frijoles y ayotes. La Compostura implica arreglar algo, reparar, curar y se trata de ceremonias en las cuales se pide buena salud, servicios comunitarios y buenas obras.

La Mano Vuelta es una costumbre mediante la cual se trabaja a cambio de un trueque 0 por reciprocidad. Cuando se casa una pareja, la comunidad entera participa en la construcción de su casa a cambio de bebidas y comida. Todos los miembros de la comunidad acuerdan trabajar un número de días en los proyectos comunitarios. Por ejemplo, durante una estadía en la zona, abrieron la carretera a una de las aldeas cercanas a Yamaranguila con piochas y palas a cambio de mano vuelta.

La Alcaldía de la Vara Alta es el consejo municipal del municipio de Yamaranguila (400 km2). Como estructura deriva de una institución política de origen español, 
pero fue apropiado por los lencas para sustituir el consejo de ancianos cuyo origen es precolombino (aparece mencionado en el Popol Vuh). La sede del Consejo es en el pueblo de Yamaranguila que cuenta con una población indígena bastante disminuida por la migración de los ladinos al pueblo. La mayoría de lencas viven en las afueras del pueblo o en las montañas cercanas. La municipalidad y la iglesia son los únicos dos edificios que todavía controlan los indígenas, y que han defendido a pesar de la presión de desalojarlos. En oportunidades varias, les han quitado la electricidad y el agua para obligarlos a marcharse.

El Consejo está conformado por un Alcalde Propietario y un Alcalde Suplente, electos cada año, un secretario que obligatoriamente debe saber leer y escribir y que es electo cada seis años; $y$ un consejo de miembros que representan cada uno de las aldeas (electos cada seis años). Las mujeres también participan (Carías y otros 1988). Este Consejo es la máxima autoridad de la comunidad y comparten las decisiones políticas. Muchos han parado en la cárcel por no asumir las decisiones impuestas por el gobierno central. Algunos miembros que no han respetado las decisiones del Consejo, han sido marginados como una forma de castigo social. Esta organización representa a los lencas de Yamaranguila en cualquier transacción que realicen con la sociedad dominante, organiza las actividades religiosas y culturales y toma las decisiones de la comunidad. Esta institución ha tenido problemas con otras organizaciones que han tratado de trabajar con ellos, pero que no han respetado las jerarquías establecidas por los lencas.

Las Vara Altas son bastones ceremoniales encomendados a ciertos ancianos del Consejo de los lencas. Chapman escribe que la Vara es un símbolo de Moisés igual que la cruz es de Cristo (1985: 66). Las cuatro Varas, según los lencas, fueron heredadas por Moisés y pasadas de mano en mano hasta nuestros días, por lo que reclaman también una herencia judía. El que posee la Vara tiene absoluta autoridad, ya que ésta representa el gran poder de la ley sagrada transmitida oralmente:

Las varas fueron halladas para que se les respete, porque ... son la primera

Consigna. De allí viene la Santa Iglesia. Las Varas son las que valen, tienen

Ley. Representan a San Francisco, La Virgen Santa Lucía, La Dolorosa, Cristo

Rey, Esquipulas, El Salvador del Mundo, San Silvestre, (etc.)... pero, primero están las Varas. Alli está la ley. Las varitas se miran como nada, pero allí está el poder. Son iguales de milagrosos, los santos y las Varas ... (op. Cit. 69, Vol. II) 
Observe el uso del diminutivo en Varitas, en conjunción con el tremendo poder simbólico que se le adjudica. Notable también es la afirmación de que los símbolos lencas son tan importantes como los católicos. En la época precolombina, los lencas usaban huesos de jaguar como símbolo de poder, muy parecido al de las Varas. El respeto a estos símbolos es necesario para obtener poder beneficioso. Existe una tensión dinámica entre el uso de estos símbolos poderosos que traían fenómenos positivos, como la lluvia; y su mal uso que traía muerte al culpable o desgracia a la comunidad. Miguel Domínguez Pérez, un informante de la comunidad de Intibucá citado por Chapman, nos advierte del peligro:

No debemos molestar a las Varas, y si nos embolamos será un poquito.

La Vara es delicada. No sólo una persona ha muerto en este oficio.

Porque se pelea con el Señor, de bolo, la Vara se enoja. Hay que pedirle perdón, ponerle una velita y un pollo ... (op. Cit. 71)

En este contexto, la preservación del ritual resulta de una importancia fundamental para los lencas y se puede esperar que se enfatice notablemente en su tradición oral. Y así ocurre, notablemente enfatizado este ritual por el consistente uso del diminutivo.

\section{EL USO DEL DIMINUTIVO}

Otra característica muy marcada de la cultura lenca es el uso del diminutivo. Su uso refuerza la identidad étnica de los lencas, y resalta la manera respetuosa para dirigirse a los elementos de vital importancia en el ceremonial lenca. Sobre esto, Cano señala que "el español andino se caracteriza por el uso amplio de diminutivos, como reflejo de los marcadores honoríficos del quechua" (2005: 1126). El uso del diminutivo no es peculiar sólo de la cultura lenca, también de otras culturas indígenas y de la cultura de Latinoamérica en general (Lapesa 1959. Cano explica que"La adopción de préstamos léxicos indígenas en el español deriva, como es obvio, de una situación de contacto de lenguas" y "la transferencia de rasgos se da. . ., el español ... . ha sido muy poroso a diversos fenómenos" de las lenguas indígena (2005:816-817).

El diminutivo en español es una herencia de los sufijos cuantitativos del latín los cuales permanecieron en la nueva lengua romance. Los sufijos eran diferentes: ellu, iccu, iculu, inu, olu e ittu y este último se desarrollo en el ito que ahora conocemos y que tiene una gran difusión en el español. Eran aplicados a sustantivos, adjetivos, adverbios y verbos. Encontramos el uso del diminutivo también en el inglés, alemán, holandés, portugués, francés y otras lenguas, pero su uso no es para 
resaltar una manera respetuosa, sino afectiva.

Según el Diccionario de la Real Academia de la Lengua Española (DRAE), diminutivo viene del latín diminutívus y tiene tres acepciones: 1) Adjetivo que tiene cualidad de disminuir o reducir a menos algo, 2) Adjetivo gramatical dicho de un sufijo que denota disminución de tamaño en el objeto designado, p. ej., en piedrecilla, o que lo presenta con intención emotiva o apelativa, p. ej., una limosnita. Se usa también con adjetivos y adverbios son significación intensiva, $p$. ej. Ahorita, y 3) la palabra formada con sufijos diminutivos.

La importancia y el valor expresivo del diminutivo viene de la relación dialéctica entre la palabra y el sufijo. Esta relación se desarrolla en dos maneras: para reforzar la palabra o para contrastarla. El sufijo puede ser también conceptual 0 axiológico. Es conceptual cuando crea un nuevo término y por lo tanto tiene un nuevo significado. Un ejemplo es maicillo (sorgo) es un término derivado de maíz, el primero es un tipo de grano y el segundo es otro tipo de grano, sin embargo debido a sus semejanzas un nuevo concepto deriva del anterior. Es axiológico cuando sutilmente cambia el valor de una palabra de positivo a negativo o de negativo a positivo. Al cambiar a una forma positiva, se convierte en un término de cariño, p. ej., Diosito de Dios. Al cambiarlo a uno negativo, se convierte en ironía, sarcasmo, p. ej., el uso de indito por parte del ladino que lo disminuye. Los lencas hacen uso del diminutivo exclusivamente para crear un sentido agregado a la palabra que modifican.

Brian Stross en una conversación personal ha sugerido que la oposición no tiene sus raíces en una valoración abiertamente relacionada al tamaño, indica que probablemente los Franciscanos y Dominicanos que convirtieron a los lencas confundieron el diminutivo del español con un afijo utilizado en las lenguas indígenas que otorgaba respeto. Esta es una investigación que pudiese realizarse en un futuro cercano por la Carrera de Letras.

Se observa un sistema de oposiciones en el uso del diminutivo por parte de los lencas en su tradición oral. En "El gigante" de Isabel Rodríguez, un gigante surge de una cueva para atacar al popular San Francisco; en "Los egueguan" por Isabel Rodríguez y en "Los segueguan" por Antonio Rodríguez las primeras personas son gigantes que comen gente, no hacen sacrificios para los dioses y son destruidos por un diluvio. 


\section{CUENTA CUENTOS}

Contar cuentos es una actividad muy importante para la comunidad lenca, se realiza en la mayoría de reuniones comunitarias, guancascos, composturas, pagos, matrimonios y funerales. Cabe aclarar que los lencas no necesitan tanto pretexto para contar sus historias. No tiene acceso a la televisión y tienen pocos radios, así que relatar cuentos es una actividad primaria para entretenerse. El autor (narrador) se coloca frente a la gente, cerca del fuego. La mayor parte de los relatos son contados por los ancianos. En línea general, son personas que han contado historias y han desarrollado una reputación por su habilidad histriónica. El público interactúa con el autor, le retroalimenta fragmentos de los relatos que ha olvidado. A menudo, El autor pide paciencia para que esperen el desenlace del relato.

Los autores conocen la cultura lenca, son "maestros de la palabra", expertos en los recursos simbólicos de su cultura (Basso 1990). Están familiarizados con las técnicas que les permiten desarrollar un relato en forma coherente. Entre los mecanismos utilizados por los autores están el paralelismo, patrones de pausas, parlamentos y repeticiones.

En este proceso, los autores lencas están reproduciendo la cultura lenca para su público, valorando los encuadres culturales, por ej., de matrimonio y de muerte. El autor está en una posición en que puede articular la lectura cultural de los fenómenos contextuales. A pesar de todo esto, sigue siendo un evento interactivo. Como señala Carbaugh,

"una cultura se evidencia en la medida en que la gente simboliza una identidad común. Esto ocurre ... en varias maneras, porque el contexto social 0 el de la comunidad está enraizado profundamente de sus raíces. Pero cada situación y cada comunidad, a través de sus patrones particulares, de sus situaciones y de sus usos comunicativos, dice algo sobre sí misma, mostrando lo que podría llamarse su identidad cultural . . .(1990:1)

Bauman señala que el evento narrativo, los eventos narrados y el "performance" están todos involucrados en el proceso creativo que tienen a mano (1990). A pesar de que la tradición oral lenca por un lado parezca conservadora, por otro representa de manera vívida los conflictos actuales. 


\section{TEORÍA Y METODOLOGÍA}

La etnografía del habla es un enfoque de la etnografía que se sitúa en un terreno entre la antropología, la lingüística, la sociología y la comunicación. Surge de las formulaciones que hace Dell Hymes a inicios de los sesentas. El modelo principal para este trabajo parte de la investigación de Joel Sherzer en "Kuna ways of speaking", en él Sherzer esboza los aspectos primordiales que son:

- Los recursos sociolingüísticos particulares de la comunidad

- El uso y explotación de estos recursos en el discurso y en la interacción social

- El patrón de los discursos con relación a interrelaciones, organización y uso social de la comunidad

- La relación de estos patrones de discurso con otros aspectos de la cultura

Este trabajo no pretende ser una etnografía completa del discurso lenca, aunque sí sirve para planear futuras investigaciones de la cultura lenca. Más que el exhaustivo y horizontal estudio que realizó Sherzer entre los kunas, éste es un análisis vertical basado en un único fenómeno, las propiedades semánticas del uso del diminutivo en la tradición oral.

Según Sherzer (1983) las investigaciones sobre "performance" implican comprender "la voz" y la "contextualización" de la misma. Significa que los hablantes utilizan mecanismos implícitos a través de los cuales señalan a sus interlocutores como debe ser interpretado aquello que dicen (Basso 1990). El uso del diminutivo expresamente sirve para seguir esta estrategia.

Las dos fuentes utilizadas son "La tradición oral de Yamaranguila", una colección de mitos y relatos recopilados por estudiantes de la Carrera de Letras de la Universidad Nacional Autónoma de Honduras para su tesis de licenciatura en 1983 y "Los hijos de Copal y Candela" escrita por Anne Chapman, recopilados entre 1965 y 1982. Ninguna de estas fuentes proporciona información detallada sobre el contexto en que estos relatos fueron recopilados, no se tuvo acceso a las grabaciones y se dependió sobre todo de las transcripciones en los dos libros para el análisis. La experiencia de otras investigaciones en la zona lenca proporcionó información adicional para enriquecer la elaboración de este trabajo.

Se utilizó un análisis de contenido pragmático cuantitativo para evaluar el uso del diminutivo por los autores lencas. Primero se contó el número de diminutivos en todos los textos y se ubicaron en categorías y subcategorías. Un ejemplo es plantita, derivado de planta, el diminutivo entra en la subcategoría de flora y en la 
categoría de medio ambiente. El propósito de esto es determinar las subcategorías utilizadas con más frecuencia en el uso del diminutivo y valorar la relevancia de estos términos en la cultura lenca. Los datos se muestran en el AnexoA.

En un segundo momento, se evaluó el uso del diminutivo a partir de un porcentaje obtenido tomando en cuenta el total de palabras en un texto dado. Los textos se clasifican en cuanto a género y por la clasificación de relato y de no relato. Los relatos incluyen los mitos, leyendas y las historias relacionadas con contar cuentos. Los no relatos incluyen los detalles de los rituales como el guancasco, matrimonio, los datos recopilados por Chapman como "Síbolos de culto y su peso emotivo" y "La actualidad: conflicto y esperanza". Los datos los brindan informantes lencas, el género es utilizado par contrastar.

Se da un traslape entre los grupos de informantes ya que Antonio Rodríguez participa en narrar: "San Antonio y su devoto", "San Isidro Patrón", "El sapo y el venado", "El hombre que sabía que los animales hablaban", "Moisés", "La Raza de Lempira", "Los Segueguan", y el no relato "La compostura de San Antonio" en "Tradición oral", luego en "Hijos del Copal y la Candela" contribuye con un relato y dos no relatos. Eleuterio Rodríguez contribuye con dos relatos en "Tradición Oral" y dos en "Hijos del Copal y la Candela".

El número total de textos en "Tradición Oral" es treinta y seis: seis relatos y dos no relatos contadas por mujeres; dieciocho relatos y ocho no relatos contados por hombres. En "Hijos del Copal y la Candela", veintiocho textos se analizaron: siete relatos y cuatro no relatos fueron narrados por mujeres y seis relatos y once no relatos fueron narrados por hombres.

\section{RESULTADOS}

Históricamente se ha utilizado la frecuencia en el análisis de contenido para indicar la importancia, la atención y el énfasis de ciertos aspectos (Krippendorf 1980). Se encontraron un total de 378 diminutivos en los sesenta y cuatro textos.

En el primer análisis el promedio de diminutivos en una subcategoría, como flora o utensilios domésticos, es de 11.09. Para hacer manejable la cantidad de información, de aquí en adelante se anotan las subcategorías cuya cantidad es $50 \%$ mayor que el promedio. Estas incluyen, en orden de frecuencia: elementos de la compostura ritual (44), animales domésticos (33), Maíz y términos relacionados (28), historia religiosa (20), y mención de las características de los miembros de la comunidad (17). Cada una de estas categorías tiene una importante relevancia para 
la cultura lenca, particularmente por la presión que han tenido en el las últimas décadas (se explica en la parte de interpretación). Los cuadros completos están al final del estudio.

En el segundo análisis no se presentan conclusiones definitivas, ya que los resultados del contraste entre los textos de acuerdo al género muestra diferentes resultados con relación al estudio del diminutivo, sin embargo, no muestran un patrón que sirva para interpretar. Apuntan más a una diferencia en las técnicas etnográficas utilizadas según las diferencias en el estilo del "performance" marcado por género o por los constructos de género. Los resultados son los siguientes:

\begin{tabular}{|c|c|c|}
\hline & Tradición Oral & Hijos del Copal \\
\hline Relatos de mujeres & $.91 \%$ & $4.88 \%$ \\
\hline No relatos de mujeres & $.5 \%$ & $3.75 \%$ \\
\hline Relatos de hombres & $1.56 \%$ & $2.00 \%$ \\
\hline No relatos de hombres & $1.73 \%$ & $2.46 \%$ \\
\hline
\end{tabular}

\section{INTERPRETACIÓN}

La interpretación pretende obtener un profundo análisis de los datos brindados por los autores lencas en los dos libros. Si se consideran los valores altamente apreciados por los lencas y se comparan con los recientes conflictos que el pueblo lenca ha sufrido con relación al gobierno, se puede comprender la marca étnica del uso del diminutivo en la reproducción social de sus valores.

El número de diminutivos encontrados en los relatos que tienen que ver con la compostura es la más alta, cuarenta y cuatro por ciento de uso de diminutivos. Esto resulta significativo ya que la religión de los indígenas de la región requería un principio de reciprocidad. Nada es gratis, uno debe trabajar para recibir los dones de los dioses. Es importante también analizar en contexto esta situación. Los sacerdotes católicos de La Esperanza y de otras regiones, les han prohibido a los lencas realizar sus ceremonias porque las consideran "paganas". En silencio pero con acierto, los lencas continúan celebrando sus rituales que son fundamentales para la sobrevivencia cultural. Aplican una "resistencia cotidiana" (Gordon en 
conversación) que persiste en el tiempo.

La cantidad de diminutivos en la subcategoría de animales domésticos es de $33 \%$, lo que demuestra la importancia de los animales en la vida lenca. Es importante señalar el anualismo en las creencias lencas. Los animales son los protectores de los seres humanos y es importante tratarlos bien.

EI Nahual protege al hombre, a la mujer, a cualquiera que sea. Entonces ... Esta familia que tiene esas ayudas, viera que se robustece! ... Ellos los Animalitos, les traen comidita luego ... y los están cuidando ...

(“ElAnual”, Isabel Rodríguez: Carías et al. 1988)

Dieciséis diminutivos se refieren a animales salvajes en los textos. Los animales domésticos también son marcados por su importancia en los rituales Lenca. Los sacrificios de los "animalitos" son prohibidos por la iglesia.

El maíz y los términos que se relacionan se marca con veintiocho diminutivos que demuestran la gran importancia del maíz en la vida lenca, no sólo como alimento, sino también como símbolo de su cultura. Un anciano lenca señala que el maíz tiene un corazoncito y un ombliguito en el fondo del grano, aunque esté seco hay vida, aunque no tenga sangre son milagrosos porque vuelven a nacer. (Carías 1988).

La chicha, bebida de maíz fermentado, es modificada en numerosas ocasiones por el diminutivo. Esta bebida es extremadamente importante en el ritual de la compostura. En "El pecado de Adán y Eva", Dios manda a Adán a buscar rapadura al infierno para poder hacer chicha, lo que nos hace interpretar el texto con sarcasmo:

Ya fue a traer el dulce Adán, y la gente ya corría a hacer bochinche, porque con el dulce hizo chicha de maíz, y los que estaban en el bochinche esos eran del diablo y los que no hacían bochinche esos eran de Dios.

(Carías etal. 1988:74)

El énfasis en los relatos religiosos y en las características de los miembros de la comunidad marca el mantenimiento de los rituales sincréticos y de la jerarquía tradicional de la "Alcaldía de la VaraAlta".

Krippendorf advierte que el análisis del contenido debe de aplicarse con una sensibilidad hacia el contexto, es decir, relacionando las figuras con la situación en cuestión (1980). Desde la perspectiva antropológica, Wolf y Wallerstein apuntan a la 
interdependencia de toda sociedad en esta era moderna, particularmente a nivel económico (Wallerstein 1982, Wolf 1982). En la década de los ochenta y de los noventa, los lencas vivieron bajo una considerable presión para abandonar sus rituales sincréticos y sus tradiciones agrícolas. Muchas organizaciones trabajaban con ellos, sin embargo, para el propósito de este estudio analizaremos dos, la Iglesia Católica y la Dirección de Recursos Naturales.

La primera institución cuyas decisiones tuvieron un impacto definitivo en la comunidad lenca, es la iglesia. Como toda institución, la iglesia en Honduras es muy compleja. Como en el resto del mundo, existen movimientos diferentes que determinan ciertas políticas y decisiones sociales. Existen grupos conservadores, como el Opus Dei, y otros grupos que apoyan los movimientos populares. En los ochentas, el Obispo era más conservador que algunos de sus subalternos que abiertamente apoyaban los movimientos de los pueblos étnicos.

En el pasado, la Iglesia ha trabajado con el gobierno para imponer ideologías hegemónicas a las poblaciones indígenas. Algunos sacerdotes apoyaban las prácticas rituales, otros las reprimían. La zona lenca ha tenido de los tipos de sacerdotes. A finales de los setenta llegó a La Esperanza (Yamaranguila depende de ésta) un sacerdote español que aumentó el grado de represión contra los rituales sincréticos de los lencas. Prohibió "los rituales paganos" y naturalmente, los lencas no siguieron sus órdenes y continuaron celebrando sus ceremonias para no peder su cultura. El sacerdote les confiscó los instrumentos para las celebraciones y les quitó la llave de la iglesia de Yamaranguila. Los lencas se opusieron y el conflicto escaló a tal grado que en junio de 1986, cuatro miembros de la Alcaldía de la Vara Alta fueron encerrados en la cárcel de La Esperanza a treinta y cinco kilómetros de allí. Fueron acusados de invadir propiedad privada porque se rehusaron a entregar las llaves de la iglesia y de entregar al sacerdote los títulos de propiedad de un pequeño potrero con doce vacas que pertenecía al patrón de Yamaranguila, San Antonio. Los lencas cultivaban el terreno y con las ganancias financiaban las celebraciones del guancasco entre Yamaranguila y La Esperanza.

La justificación del sacerdote era de que si la tierra pertenecía al Santo Patrón, entonces era propiedad de la iglesia. Otro argumento era que los rituales paganos no podían celebrarse en territorio de la iglesia. El sacerdote se oponía al consumo de chicha, elemento esencial en el ritual lenca. Sin embargo, instaló una productora de vino de papa.

Es adecuado señalar que el conflicto de la iglesia con la comunidad está obligando a que se dividan los miembros entre los tradicionalistas y los seguidores de la 
modernidad. Esto afecta a la comunidad y los hace compactarse para responder adecuadamente. Esta situación por otra parte, promueve la integración de la comunidad para reproducir su propia cultura en beneficio de las generaciones más jóvenes. Los ancianos tienen una gran responsabilidad en educar a los jóvenes sobre las tradiciones lencas. Es posible que dado el conflicto muchos miembros no participen colectivamente en la comunidad, pero también es posible que de esta situación resulte una mayor cohesión como una contra reacción.

Con esta información, no resulta extraña la necesidad de marcadores étnicos que agreguen valor a los aspectos de la compostura, incluidos los animales domésticos (sacrificados). Todo esto toma una dimensión adicional, ya que estas entrevistas y las grabaciones se hicieron básicamente entre el comienzo y mediados de los ochenta cuando se dio la mayor crisis con la iglesia. Una inferencia lógica es que el uso del diminutivo que marca más los elementos más valorados de su cultura corresponde a fenómenos que los ancianos trataban de preservar frente a las presiones externas. Sus narrativas demuestran como sus "performances" reflejaban dicha preocupación.

La segunda institución de la cual analizaremos su impacto es la Dirección de Recursos Naturales, que es una de las organizaciones más antiguas en operar en la zona lenca. Fue creada en 1940 para desarrollar los recursos naturales de Honduras. El país, con su tradición agrícola, necesitaba una institución centralizada para coordinar el desarrollo agrícola. La presencia permanente de la Dirección en la zona lenca es reciente. Los promotores (extensionistas) llegaron en los ochenta y trabajan en áreas específicas: agricultura, ganado y actividades de amas de casa.

A menudo los extensionistas se encuentran entre la espada y la pared, porque las decisiones que se toman no están articuladas con otras instituciones y hacen fracasar cualquier intento de mejorar las condiciones socioeconómicas de los pueblos étnicos. Por ejemplo, se estimula la producción de ciertas cosechas; pero, al momento de vender el producto si las carreteras no están en optimas condiciones, no se puede sacar la cosecha de los alejados y aislados valles. Estos fracasos han hecho que los pueblos tengan una gran desconfianza del gobierno.

Las anteriores descripciones nos muestran como el impacto de dos instituciones para la modernización del Estado, han tenido impactos negativos en la vida y en la integración de la comunidad lenca. Aunque reconocen la labor de los extensionistas en algunos aspectos, también señalan la falta de una verdadera articulación para que el desarrollo ocurra. Por lo que están camino a convertirse en fuerza de trabajo asalariada para las compañías agroindustriales del cinturón de desarrollo en 
Honduras.

El primer análisis cuantitativo sustenta la tesis de que el uso del diminutivo marca los conceptos emergentes de la narrativa lenca. El segundo análisis en que se contrasta el género no es concluyente. Describe únicamente los diferentes estilos de grabación y de transcribir datos. Algunas premisas tentativas podrían lanzarse, como el hecho de que la autoría entre los lencas es posible para las mujeres o los hombres. Como no se hizo una diferenciación entre los espacios públicos y/o privados, no es posible inferir nada. Lo que sí se puede señalar es que la Dra. Anne Chapman recogió en sus textos más diminutivos que los que recogieron los estudiantes de la Carrera de Letras de laUNAH.

\section{CONCLUSIONES}

Los lencas de Yamaranguila, a nivel conciente o inconsciente, utilizan el diminutivo para marcar los símbolos importantes de su cultura. Los conceptos emergentes se contrastan en oposición a los fenómenos fuera de la comunidad lenca. Un "nosotros" y un "ellos" está claramente articulado en la realidad que presentan.

En vista de las oprobiosas condiciones de vida de los lencas, no es sorprendente para nada encontrar marcadores para aspectos emergentes en los textos. Ellen Basso, citando a Susan Paulson, plantea que los discursos ambiguos, de los que nos hemos apropiado en este trabajo, pueden ser considerados como discursos de resistencia.

... los discursos ambiguos como mecanismos de sobre vivencia ante el contacto con otra cultura se vuelven claros, la metodología de análisis de los discursos y de los textos revela que existen mensajes escondidos trasmitidos durante tiempos de terror, cuando la gente no puede expresar abiertamente sus dudas sobre la ideología impuesta, de tal forma que la resistencia a los que ostentan el poder se teje en una secretividad extremadamente sutil ... (1990)

Las conclusiones que se obtuvieron del análisis de los datos en un sentido no son concluyentes, se necesitaría realizar los patrones de habla de los lencas en un período más amplio para compararlos con las transcripciones de los ochenta. Sin embargo, el concluir que el uso del diminutivo representa un código cultural de los lencas es irrefutable. Un estudio más amplio del diminutivo describiendo el porqué, el cuándo, el dónde, el qué y el quién sería útil para el análisis de las perspectivas culturales de los lencas. 


\section{BIBLIOGRAFÍA}

Barry, Tom y Deb Preusch. 1986. The Central American Fact Book. New York: Grove Press.

Basso, Ellen. 1990. Native Latin American Cultures Through Their Discourse.

Bloomington: Folklore Institute Indiana University.

Basso, Keith. 1991. Portraits of the "Whiteman". 12 ed. New York: Cambridge University Press.

Bauman, Richard. 1990. Story Performance and Event. Cambridge: Oxford University Press.

Bauman, Richard.1992. Folklore, Cultural Performances, and Popular Entertainments. Oxford: Oxford University Press.

Blas Arroyo, José Luis. 2005. Sociolingüística del Español: desarrollos y perspectivas en el estudio de la lengua española en contexto social. Madrid: Cátedra.

Campbell, Lyle Richard. "The Last Lenca". International Journal of American Linguists. 42 (1) 73-78. 1978.

Cano, Rafael. 2005. Historia de la Lengua Española. Barcelona:Ariel.

Carías, Claudia y otros. 1988. Tradición oral lenca de Yamaranguila. Tegucigalpa: Guaymuras.

Carmack, Robert. 1934. The Quiche Mayas of Utatlan: The Evolution of a Highland Kingdom. Norman: University of Oklahoma Press.

Chapman, Anne. 1985. Los hijos del Copal y la Candela. México: Imprenta Universitaria UNAM.

Chávez, Manuel. 1992. Como subsisten los campesinos. Tegucigalpa: Guaymuras.

Eco, Humberto. 1973. Segno. Milano: Instituto Editoriale Internazionale.

Finnegan, Ruth. 1970. Oral Literatura in Africa. Oxford: Claredon Press.

1992. Oral Tradition and the Verbal Arts. London: Routledge.

Gossen, Harry. 1986. Symbol and Meaning beyond the Closed Community:

Esay in Mesoamerican Ideas. Albany: Institute for Mesoamerican Studies.

Heller, Agnes. 2002. Sociología de la vida cotidiana. Barcelona: Península.

Hymes, Dell. 1981. "In Vain I tried to tell you": Essays in Native American

Ethnopoetics. Philadelphia: University of Philadelphia Press.

Krippendorf, Klaus. 1980. Introduction to Content Analysis: Its Use and Methodology. Boulder: Westview Press.

LAB. 1985. Honduras State for Sale. London: Latin American Bureau.

Lapesa, Rafael. 1959. Historia de la Lengua Española. 4 ed. Madrid: Gredos.

León Portilla, Miguel. 1988. Time and Reality in the Thought of the Mayas. $2 \mathrm{ed}$. 
Translated by Charles Boiles, Fernando Horcasitas and the Author. Norman: University of Oklahoma Press.

Nañez Fernández,Emilio. 1973. El Diminutivo. Madrid: Gredos.

Sherzer, Joel. 1983. Kuna Ways of Speaking. Austin: University of Texas Press.

Sherzer, Joel and Greg Urban. 1984. Native South American Discourses. Berlin:

Mouton de Gruyter.

Tedlock, Dennis. 1972. Finding the Center: Narrative Poetry of the Zuni Indians. New York: The Dial Press.

Ungar, Steven and Betty McGraw. 1989. Signs in Culture: Roland Barthes

Today. lowa: University lowa Press.

Wallerstein y Hopkins. 1982. World System Analysis: Theory and Methodology. London: Sage.

Wolf, Eric. 1962. Peuples et Civilisations de L'Amerique Central. Traduit de lánglais par Bernard de Zelicourt. Paris : Payot.

Peasants. New Jersey : Prentice Hall. 1966.

Europe and the People without History. 2 ed. California: University Press Ltd. 1982.

\section{Anexos}

Uso del diminutivo en la tradición oral

Tradición oral indígena de Yamaranguila: Carías y otros Los hijos del Copal y la Candela: Anne Chapman

\begin{tabular}{|l|c|c|c|}
\hline \multicolumn{1}{|c|}{ CATEGORIÁ } & HCC & TOIY & TOTALES \\
\hline SALUD & 11 & 1 & 12 \\
\hline NOMBRES & 2 & 3 & 5 \\
\hline RELIGIÓN, IDEAS, CREENCIAS & & & \\
\hline $\begin{array}{l}\text { A. ELEMENTOSDELRITUALDELACOMPOSTURA } \\
\text { (fresquito, referido a la chicha ocho veces) }\end{array}$ & 24 & 20 & 44 \\
\hline B. ELEMENTOS DE LA IGLESIA & 4 & 2 & 6 \\
\hline C. RELACIONADOS CON LA MUERTE & 1 & 3 & 4 \\
\hline D. PERSONAJES RELIGIOSOS & 1 & 3 & 4 \\
\hline E. HISTORIA RELIGIOSA & 18 & 2 & 20 \\
\hline F. ANIMALES RELACIONADOS CON SANTOS & 8 & 3 & 11 \\
\hline G. ORGANIZACIÓN DEL RITUAL & 11 & 2 & 13 \\
\hline VIDA COTIDIANA & & & 11 \\
\hline A. ASPECTOS DEL CUERPO HUMANO & 4 & 7 & 12 \\
\hline B. UTENSILIOS DOMESSTICOS & 4 & 8 & 7 \\
\hline C. VESTIMENTA & 1 & 6 & 9 \\
\hline D. OBJETOS DE CASA & 0 & 9 & 9 \\
\hline
\end{tabular}




\begin{tabular}{|l|c|c|c|}
\hline \multicolumn{1}{|c|}{ CATEGORÍA } & HCC & TOIY & TOTALES \\
\hline e. COMIDA PREPARADA & 1 & 8 & 9 \\
\hline f. MATERIALES CONBUSTIBLES & 0 & 5 & 5 \\
\hline g. Subsistencia & 0 & 1 & 1 \\
\hline RELACIONES SOCIALES & & & \\
\hline A. vida social & 2 & 8 & 10 \\
\hline B. relaciones familiares & 5 & 9 & 14 \\
\hline C. personas ancianas & 1 & 13 & 14 \\
\hline D. Niños & 2 & 6 & 8 \\
\hline E. gente de la comunidad & 1 & 3 & 4 \\
\hline F. origen étnico & 0 & 13 & 13 \\
\hline G. características de la gente de la comunidad & 1 & 17 & 7 \\
\hline MEDIO AMBIENTE & & & \\
\hline A. animales domésticos & 17 & 16 & 33 \\
\hline B. animales silvestres & 1 & 13 & 14 \\
\hline C. Flora & 2 & 11 & 13 \\
\hline D. descripciones geográficas & 1 & 6 & 7 \\
\hline E. nombres de lugares & 1 & 4 & 5 \\
\hline PRODUCCIÓN Y CONSUMO & & & \\
\hline A. aspectos relacionados con la tierra & 3 & 7 & 10 \\
\hline B. maíz y términos relacionados & 11 & 17 & 28 \\
\hline C. implementos de trabajo & 2 & 7 & 9 \\
\hline TIEMPO, ESPACIO, CANTIDAD & & & \\
\hline A. referencia a la noche & 0 & 1 & 1 \\
\hline B. aspectos de cantidad & 2 & 4 & 6 \\
\hline C. indicaciones de tiempo & 2 & 5 & 7 \\
\hline D. indicaciones de lugar & 0 & 6 & 6 \\
\hline TOTAL & 129 & 249 & 378 \\
\hline
\end{tabular}


El uso del diminutivo por mujeres

Tradición oral Lenca de Yamaranguila

\begin{tabular}{|l|l|c|c|c|}
\hline \multicolumn{1}{|c|}{ Autora } & \multicolumn{1}{c|}{$\begin{array}{c}\text { Nombre del } \\
\text { texto }\end{array}$} & $\begin{array}{c}\text { No. de } \\
\text { palabras }\end{array}$ & $\begin{array}{c}\text { No. de } \\
\text { diminutivos }\end{array}$ & Porcentaje \\
\hline Francisca Lemus & $\begin{array}{c}\text { - El pecado de } \\
\text { Adán y Eva }\end{array}$ & 120 & 5 & $0.4 \%$ \\
\hline Filomena Vásquez & $\begin{array}{l}\text { - Como apareció } \\
\text { el maíz }\end{array}$ & 410 & 7 & $2.0 \%$ \\
\cline { 2 - 5 } & - La madrina & 400 & 4 & $1.0 \%$ \\
\hline Martha Beatriz Reyes & $\begin{array}{l}\text { - Compostura } \\
\text { de San Antonio }\end{array}$ & 190 & 0 & $0.0 \%$ \\
\cline { 2 - 5 } & - El Semejante & 280 & 0 & $0.0 \%$ \\
\hline Dolores Reyes & - Los velorios & 650 & 8 & $1.0 \%$ \\
\hline Irene Manueles & - El gigante & 380 & 4 & $1.0 \%$ \\
\hline Luz Argentina Guevara & $-\begin{array}{c}\text { El levantamiento } \\
\text { del anima }\end{array}$ & 490 & 3 & $1.0 \%$ \\
\hline
\end{tabular}

\section{El uso del diminutivo por mujeres}

Los hijos del Copal y la Candela

\begin{tabular}{|l|l|c|c|c|}
\hline Autora & $\begin{array}{c}\text { Nombre del } \\
\text { texto }\end{array}$ & $\begin{array}{c}\text { No. de } \\
\text { palabras }\end{array}$ & $\begin{array}{c}\text { No. de } \\
\text { diminutivos }\end{array}$ & Porcentaje \\
\hline Dolores Reyes & $\begin{array}{l}\text { - La aparición de San } \\
\text { Francisco de Asís }\end{array}$ & 50 & 3 & $6.0 \%$ \\
\cline { 2 - 5 } & $\begin{array}{l}- \text { San Silvestre del } \\
\text { Monte }\end{array}$ & 70 & 1 & $1.4 \%$ \\
& $\begin{array}{l}\text { - Ceremonia de la } \\
\text { Auxiliaría de la } \\
\text { Vara Alta }\end{array}$ & 35 & 1 & $2.0 \%$ \\
\hline $\begin{array}{l}- \text { Símbolos de culto } \\
\text { y peso emotivo }\end{array}$ & 140 & 4 & $2.8 \%$ \\
\hline $\begin{array}{l}- \text { Ceremonias en la } \\
\text { Vara Alta de Moisés }\end{array}$ & 50 & 2 & $4.0 \%$ \\
\hline Manuela Gómez & $\begin{array}{l}- \text { La arriaga de San } \\
\text { Francisco de Asís }\end{array}$ & 200 & 1 & $0.5 \%$ \\
\hline$-\begin{array}{l}\text { Símbolos de culto y } \\
\text { peso emotivo }\end{array}$ & 80 & 1 & $1.25 \%$ \\
\hline Margarita Lemus & $\begin{array}{l}- \text { Cruces y patrones } \\
\text { de las aldeas }\end{array}$ & 30 & 1 & $3.3 \%$ \\
\hline
\end{tabular}




\begin{tabular}{|l|c|c|c|c|}
\hline \multicolumn{1}{|c|}{ Autora } & \multicolumn{1}{|c|}{$\begin{array}{c}\text { Nombre del } \\
\text { texto }\end{array}$} & $\begin{array}{c}\text { No. de } \\
\text { palabras }\end{array}$ & $\begin{array}{c}\text { No. de } \\
\text { diminutivos }\end{array}$ & Porcentaje \\
\hline Arcadia Bautista & $\begin{array}{c}\text { - La Virgen de los } \\
\text { Remedios castiga }\end{array}$ & 240 & 7 & $2.9 \%$ \\
\hline Beatriz Reyes Cantarero & $\begin{array}{c}\text { - Celebración de la } \\
\text { Auxiliaría de la Vara } \\
\text { Alta de Moisés }\end{array}$ & 59 & 4 & $8.0 \%$ \\
\hline Mujer de Yamaranguila & $\begin{array}{c}\text { - Actualidad, conflicto } \\
\text { y esperanza }\end{array}$ & 190 & 3 & $1.5 \%$ \\
\hline
\end{tabular}

\section{El uso del diminutivo por hombres Tradición oral}

\begin{tabular}{|c|c|c|c|c|}
\hline Autor & $\begin{array}{l}\text { Nombre del } \\
\text { texto }\end{array}$ & $\begin{array}{l}\text { No. de } \\
\text { palabras }\end{array}$ & $\begin{array}{c}\text { No. de } \\
\text { diminutivos }\end{array}$ & Porcentaje \\
\hline Juan López & $\begin{array}{l}\text { - Oración para la } \\
\text { compostura en el } \\
\text { cambio de mayordo- } \\
\text { mía }\end{array}$ & 550 & 7 & $0.78 \%$ \\
\hline Bautista Porfirio & $\begin{array}{l}\text { - Oración para enco- } \\
\text { mendar a los jóvenes }\end{array}$ & 75 & 4 & $5.0 \%$ \\
\hline \multirow[t]{8}{*}{ Antonio Rodríguez } & $\begin{array}{l}\text { - San Antonio y su } \\
\text { devoto }\end{array}$ & 1240 & 4 & $0.3 \%$ \\
\hline & - El sapo y el venado & 450 & 0 & $0.0 \%$ \\
\hline & - San Isidro Patrón & 1620 & 3 & $0.1 \%$ \\
\hline & $\begin{array}{l}\text { - El hombre que sabía } \\
\text { que los animales } \\
\text { hablaban }\end{array}$ & 780 & 3 & $0.3 \%$ \\
\hline & - Moisés & 50 & 0 & $0.0 \%$ \\
\hline & - La raza de Lempira & 470 & 4 & $1.0 \%$ \\
\hline & - Los Segueguan & 250 & 0 & $0.0 \%$ \\
\hline & $\begin{array}{l}\text { - La compostura de } \\
\text { San Antonio }\end{array}$ & 140 & 4 & $3.0 \%$ \\
\hline \multirow[t]{3}{*}{ Eleuterio Rodríguez } & $\begin{array}{l}\text { - La composición de } \\
\text { pueblo de Yamaran- } \\
\text { guila }\end{array}$ & 1130 & 14 & $1.0 \%$ \\
\hline & - Lempira & 480 & 4 & $1.0 \%$ \\
\hline & $\begin{array}{l}\text { - El zorro dueño del } \\
\text { maíz }\end{array}$ & 360 & 0 & $0.0 \%$ \\
\hline Leonardo Manueles & $\begin{array}{l}\text { - El alcalde que no } \\
\text { sabía leer }\end{array}$ & 1080 & 1 & $0.0 \%$ \\
\hline
\end{tabular}




\begin{tabular}{|l|l|c|c|c|}
\hline \multicolumn{1}{|c|}{ Autor } & \multicolumn{1}{|c|}{$\begin{array}{c}\text { Nombre del } \\
\text { texto }\end{array}$} & $\begin{array}{c}\text { No. de } \\
\text { palabras }\end{array}$ & $\begin{array}{c}\text { No. de } \\
\text { diminutivos }\end{array}$ & Porcentaje \\
\hline \multirow{5}{*}{ Isabel Rodríguez } & - Los Guancascos & 720 & 4 & $0.5 \%$ \\
\cline { 2 - 5 } & - El matrimonio & 720 & 4 & $0.5 \%$ \\
\hline & $\begin{array}{l}\text { - La formación de } \\
\text { mundo }\end{array}$ & 390 & 10 & $3.0 \%$ \\
\cline { 2 - 5 } & - El granito de maíz & 180 & 9 & $5.0 \%$ \\
\cline { 2 - 5 } & - El maíz común & 190 & 6 & $3.0 \%$ \\
\cline { 2 - 5 } & - El nahual & 140 & 2 & $1.0 \%$ \\
\cline { 2 - 5 } & - Los matrimonios & 830 & 11 & $1.0 \%$ \\
\hline Roberto Vásquez & $\begin{array}{l}\text { - Los árboles echaban } \\
\text { sangre }\end{array}$ & 270 & 6 & $2.0 \%$ \\
\hline Santiago Vásquez & - El Guancasco & 390 & 3 & $0.8 \%$ \\
\hline
\end{tabular}

\section{El uso del diminutivo por hombres Los hijos del Copal y la Candela}

\begin{tabular}{|c|c|c|c|c|}
\hline Autor & $\begin{array}{l}\text { Nombre del } \\
\text { texto }\end{array}$ & $\begin{array}{l}\text { No. de } \\
\text { palabras }\end{array}$ & $\begin{array}{c}\text { No. de } \\
\text { diminutivos }\end{array}$ & Porcentaje \\
\hline Tomás Vásquez & - Los Guancascos & 120 & 4 & $3.3 \%$ \\
\hline \multirow[t]{2}{*}{ Antonio Rodríguez } & $\begin{array}{l}\text { - Evocaciones Mitoló- } \\
\text { gicas y mitos }\end{array}$ & 120 & 2 & $1.6 \%$ \\
\hline & $\begin{array}{l}\text { - Actualidad, conflicto } \\
\text { y esperanza }\end{array}$ & 80 & 1 & $1.25 \%$ \\
\hline \multirow[t]{2}{*}{ Tiburcio Rodríguez } & $\begin{array}{l}\text { - Símbolos de culto y } \\
\text { peso emotivo }\end{array}$ & 80 & 3 & $3.75 \%$ \\
\hline & $\begin{array}{l}\text { - Adán y Eva hacen } \\
\text { la primera venera- } \\
\text { ción a la tierra }\end{array}$ & 530 & 14 & $2.6 \%$ \\
\hline \multirow[t]{2}{*}{ Senforiano Rodríguez } & $\begin{array}{l}\text { - Las aves del corral } \\
\text { hicieron las primeras } \\
\text { leyes }\end{array}$ & 260 & 1 & $0.4 \%$ \\
\hline & $\begin{array}{l}\text { - La aparición de San } \\
\text { Francisco de Asís }\end{array}$ & 160 & 2 & $1.25 \%$ \\
\hline Porfirio Aguilar & $\begin{array}{l}\text { - Símbolos de culto y } \\
\text { su peso emotivo }\end{array}$ & 80 & 1 & $1.25 \%$ \\
\hline Don Silverio & $\begin{array}{l}\text { - Actualidad, conflicto } \\
\text { y esperanza }\end{array}$ & 80 & 1 & $1.25 \%$ \\
\hline Don Delfino & - Rito en casa & 210 & 1 & $0.4 \%$ \\
\hline Don Artemio & $\begin{array}{l}\text { - Actualidad, conflicto } \\
\text { y esperanza }\end{array}$ & 80 & 1 & $1.75 \%$ \\
\hline
\end{tabular}




\begin{tabular}{|c|c|c|c|c|}
\hline Autor & $\begin{array}{l}\text { Nombre del } \\
\text { texto }\end{array}$ & $\begin{array}{l}\text { No. de } \\
\text { palabras }\end{array}$ & $\begin{array}{c}\text { No. de } \\
\text { diminutivos }\end{array}$ & Porcentaje \\
\hline \multirow[t]{2}{*}{ Eleuterio Rodríguez } & - La gente antes & 150 & 2 & $1.3 \%$ \\
\hline & $\begin{array}{l}\text { - Adán y Eva hacen } \\
\text { la primera venera- } \\
\text { ción a la tierra }\end{array}$ & 150 & 2 & $1.3 \%$ \\
\hline Hipólito Lara & $\begin{array}{l}\text { - Símbolos de culto y } \\
\text { su peso emotivo }\end{array}$ & 60 & 5 & $8.3 \%$ \\
\hline \multirow[t]{2}{*}{ Gonzalo García } & $\begin{array}{l}\text { - Otra bendición } \\
\text { después que sacri- } \\
\text { ficaron los patos }\end{array}$ & 280 & 5 & $1.8 \%$ \\
\hline & $\begin{array}{l}\text { - Símbolos de culto } \\
\text { y su peso }\end{array}$ & 110 & 4 & $3.6 \%$ \\
\hline
\end{tabular}

